\title{
EL SÍNDROME DE ASPERGER Y SU CLASIFICACIÓN
}

\author{
Marco Antonio Zúñiga Montero \\ Director Departamento de Anatomía, Escuela de Medicina de la \\ Universidad de Costa Rica \\ San José, Costa Rica
}

Recibido 13-III-2009 • Aceptado 30-V-2009 • Corregido 8-VI-2009

\begin{abstract}
Resumen: El artículo trata sobre el Síndrome de Asperger, que es un trastorno generalizado del desarrollo. Su causa primaria parece ser genética y recientemente fue reconocido como tal por la comunidad científica. Debido a sus características, esta enfermedad es poco conocida por la población e, incluso, por ser tan reciente su descubrimiento, muchos profesionales de las áreas educativa, social y médica no están suficientemente informados al respecto. Muchas familias costarricenses lo presentan. Tradicionalmente, este síndrome se incluye dentro del espectro autista; sin embargo, está contemplado en el DSM IV como Trastorno de Asperger y en el CIE10 como Síndrome de Asperger, que lo clasifica como una entidad clínica diferente del autismo.
\end{abstract}

Palabras clave: Historia, desarrollo científico, educación, Síndrome de Asperger.

\begin{abstract}
The article presents the Syndrome of Asperger that is a development generalized disorder. Its primary cause seems to be genetic and recently it was recognized as so by the scientific community. Due to its characteristics, this disease is little known by the population and even, for being of so recent discovery, many professionals of the educative, social and medical areas are not sufficiently informed on this subject. Many Costa Rican families present it. Traditionally, this syndrome is included within the autistic spectrum; nevertheless, it is contemplated in DSMIV as the Disorder of Asperger and in the CIE-10 as the Syndrome of Asperger. That classifies it as a clinical entity different from autism.
\end{abstract}

Key words: History, scientific development, education, Asperger's Syndrome.
El Síndrome de Asperger (SA) fue descrito en 1944 por el médico pediatra austriaco Hans Asperger. Este hecho casi coincidió, cronológicamente, con la publicación en 1943 del doctor Leo Kanner sobre los trastornos autísticos del contacto afectivo, cuyo trabajo dio origen a las ideas modernas del trastorno autista. Kanner dirigió el Servicio de Psiquiatría Infantil del Hospital John Hopkins de Baltimore. El Dr. Kanner nació en Austria y emigró a los Estados Unidos en 1924.

Sin que ninguno de los dos supiera del otro, Kanner publicó en inglés la historia clínica de once niños con trastornos autísticos, y Asperger, en alemán, la de cuatro niños con psicopatía autística que, presumiblemente, tenían conductas autistas poco severas y un coeficiente normal de inteligencia.

Tanto las descripciones de Asperger como las de Kanner se centran en aspectos muy peculiares de la conducta infantil. Ambos autores hicieron referencia a pacientes con alteraciones en el comportamiento social, el lenguaje y las habilidades cognitivas. A pesar de que posiblemente Asperger no conocía el trabajo de Kanner, publicado con el título de Alteraciones 
autísticas del contacto afectivo, utilizó el término de psicopatía autística. De esta manera, ambos autores destacaban el aislamiento que manifestaban sus pacientes.

Asperger, junto con una colaboradora suya, la hermana Viktorine, había desarrollado en el Hospital Universitario Infantil de Viena un ambicioso programa de rehabilitación, basado en la terapia del lenguaje, la representación teatral y la educación física. Desgraciadamente, la guerra terminó con la vida de la hermana y las bombas aliadas destruyeron el hospital. A pesar de ello, Asperger continuó trabajando como pediatra, hasta fallecer el 21 de octubre de 1980, poco antes de que su trabajo fuera aceptado por la comunidad médica internacional.

Pasaron casi cuarenta años desde la publicación del Dr. Asperger, y en 1981 la psiquiatra inglesa Dra. Lorna Wing publica, en la revista Psychological Medicine, el trabajo denominado "Asperger's Syndrome: A clinical account", cuyo título mencionaba por primera vez en la literatura científica el nombre con el que actualmente se conoce este trastorno del desarrollo. El trabajo de la Dra. Wing reinició la investigación internacional sobre el síndrome al que el doctor Asperger había dado nombre - sin haberse enterado-. A partir de esta publicación (Wing, 1981), se ha manifestado la importancia de este síndrome, debido a la conducta social de las personas que lo presentan.

El concepto de espectro autista tiene su origen en un estudio realizado por Lorna Wing y Judith Gould en 1979 (Wing y Gould, 1979) en una barriada de Londres. Para ellas, los rasgos del autismo no solo estaban presentes en personas autistas, sino también en otros cuadros de trastornos del desarrollo, pues, de acuerdo con el grado de afectación de estas personas, no cumplen los criterios estrictos del autismo; no obstante, requieren de un tratamiento similar.

La publicación original de Asperger fue traducida al inglés en 1991 por Frith. Ella puede constituir una herramienta útil de consulta para estudiar las observaciones originales de Kanner y Asperger, y comprender con más profundidad los puntos controvertidos.

En 1994, en el CIE-10 que es la décima revisión de la "Clasificación estadística internacional de enfermedades y problemas de salud", de la Organización Mundial de la Salud [OMS], esta afección aparece en la sección de Trastornos generalizados del desarrollo como Síndrome de Asperger (p. 40):

Clasificación de trastornos mentales CIE-10

F84 Trastornos generalizados del desarrollo

F84.0 Autismo infantil

F84.1 Autismo atípico

F84.2 Síndrome de Rett

F84.3 Otros trastornos desintegrativos de la infancia

F84.4 Trastorno hipercinético con retraso mental y movimientos estereotipados

F84.5 Síndrome de Asperger

F84.8 Otros trastornos generalizados del desarrollo

F84.9 Trastornos generalizados del desarrollo sin especificación.

En 1994, en los Estados Unidos, la Asociación Americana de Psiquiatría (American Psychiatric Association-APA) clasificó por primera vez el trastorno de Asperger en el Manual diagnóstico y estadístico de trastornos mentales DSM IV [Diagnostic and Statistical Manual of Mental Disorders DSM IV], en la categoría de "Trastornos generalizados del desarrollo", los cuales se definen por las conductas y manifestaciones observables. En este manual aparece este término, no como síndrome, sino como trastorno. Ambos términos describen la misma condición (APA, 1994).

En el Manual DSM IV, el Asperger es uno de los cinco trastornos definidos bajo la categoría de "Trastornos generalizados del desarrollo". Los otros cuatro trastornos son: trastorno del autismo, trastorno de Rett, trastorno de la desintegración infantil, y trastorno generalizado del desarrollo no especificado.

El Síndrome de Asperger (SA) es un trastorno del desarrollo neurológico 
que afecta el funcionamiento social y el espectro de actividades e intereses. Está vinculado a una disfunción de diversos circuitos cerebrales (Artigas, 2007).

Cada niño, niña o persona adulta que presenta el Síndrome de Asperger, manifiesta diferentes características de la personalidad y/o dificultades en la interacción social durante su vida, que en algunos casos pasan inadvertidas. Aunque las personas con este síndrome tienen un aspecto físico y una capacidad intelectual normales, enfrentan dificultades -que varían de leves a graves- originadas durante su desarrollo, como dificultades en la interacción social y en la comunicación verbal y no verbal, rutinas repetitivas, poca flexibilidad de pensamiento e intereses específicos (Barquero, 2007).

\section{Conclusiones}

El Síndrome de Asperger ha tenido una importante evolución a lo largo del tiempo, pues ya en la década de los años cuarentas se describieron sus principales rasgos en pacientes. Sin embargo, debido a la divulgación que ha tenido en los últimos años, se ha podido investigar y comprender más las características del síndrome. Así, ha quedado claro que el Síndrome de Asperger tiene un reconocimiento oficial y está incluido en los manuales internacionales de clasificación psiquiátrica CIE-10 y DSM-IV, como un trastorno generalizado del desarrollo, diferente del trastorno autista, el cual se encuentra también dentro de esta clasificación.

Como es bien sabido, la "Ley de Igualdad de Oportunidades para las Personas con Discapacidad", $\mathrm{N}^{\circ} 7600$, vigente desde el 29 de mayo de 1996, establece que el Estado costarricense debe conceder igualdad de oportunidad en el acceso a la educación a todas las personas con discapacidad, cualquiera que sea su limitación o enfermedad, física, mental o sensorial, en procura de facilitarles un entorno adecuado, a efecto de disminuir el impacto de la dolencia que los esté afectando (Costa Rica, Leyes y Decretos, 1998).

En tales circunstancias, se considera que el Síndrome de Asperger, una entidad clínica diferente del autismo, podría incorporarse formalmente en el proceso de adecuaciones curriculares, para que ningún estudiante, afectado por dolencias poco conocidas, resulte injustamente excluido del trato especial que su problema de salud haga necesario, y para que los estudiantes puedan tener un sistema educativo integral, solidario y de calidad, que garantice la igualdad de oportunidades y actúe desde el respeto a la diversidad.

\section{Referencias bibliográficas}

American Psychiatric Association [APA]. (1994). Diagnostic and statistical manual of mental disorders [Diagnóstico y manual estadístico de desórdenes mentales] (4th. ed.). Washington DC: APA.

Artigas, J. (2007). Aspectos neurobiológicos del Síndrome de Asperger. Un acercamiento al Síndrome de Asperger: Una guía teórica y práctica. Sevilla: Asociación de Asperger Andalucía.

Asperger, H. (1944). Die autistischen psichopathen im kindersalter [Psicopatía autística en la infancia]. Archiv für Psychiatrie und Nervenkrankheiten, 117, 76-136.

Barquero, M. (2007). Síndrome de Asperger. Guía para padres de familia y educadores. San José: Vicerrectoría de Acción Social, Universidad de Costa Rica.

Costa Rica, Leyes y Decretos. (1996, 29 de mayo). Ley $\mathrm{N}^{\circ}$ 7600. Igualdad de oportunidades para las personas con discapacidad. Diario Oficial La Gaceta, pp. 1-8. 
Frith, U. (1991). Autism and Asperger's Sindrome [Austismo y Síndrome de Asperger]. Londres: Cambridge University Press.

Kanner, L. (1943). Autistic disturbances of affective contact [Alteraciones autísticas del contacto afectivo]. The Nervous Child, 2, 217-250.

Organización Mundial de la Salud [OMS]. (1994). CIE-10. Trastornos mentales $y$ del comportamiento. Descripciones clínicas y pautas para el diagnóstico. Madrid: Mediator.
Wing, L. (1981). Asperger's Syndrome: A clinical account [Síndrome de Asperger: Un análisis clínico]. Psychological Medicine, 11, 115-130.

Wing, L. y J., Gould. (1979). Severe impairments of social interaction and associated abnormalities in children: Epidemiology and classification [Deterioro severo de la interacción social y otros trastornos asociados con niños: Epidemiología y clasificación]. Journal of Autism and Developmental Disorders, 9, 11-29. 